\title{
Civilisations
}

Revue internationale d'anthropologie et de sciences

humaines

$48 \mid 2001$

La question de l'islam et de l'Etat à l'aube du XXIe siècle

\section{Les projets de constitution palestinienne (1993-2000) : L'islam comme élément de souveraineté}

Jean-François Legrain

\section{(2) OpenEdition}

Journals

Édition électronique

URL : http://journals.openedition.org/civilisations/3468

DOI : $10.4000 /$ civilisations.3468

ISSN : 2032-0442

Éditeur

Institut de sociologie de l'Université Libre de Bruxelles

Édition imprimée

Date de publication : 31 août 2001

Pagination : 121-137

ISSN : 0009-8140

\section{Référence électronique}

Jean-François Legrain, « Les projets de constitution palestinienne (1993-2000) : L'islam comme élément de souveraineté », Civilisations [En ligne], 48 | 2001, mis en ligne le 01 août 2004, consulté le 30 avril 2019. URL : http://journals.openedition.org/civilisations/3468 ; DOI : 10.4000/ civilisations.3468 


\title{
LES PROJETS DE CONSTITUTION PALESTINIENNE (1993-2000): L'ISLAM COMME ÉLÉMENT DE SOUVERAINETÉ
}

\author{
Jean-François LEGRAIN
}

\section{INTRODUCTION}

Ces trente dernières années, toute une littérature a été produite à propos du «conflit israéloarabe» et de «la question palestinienne», à la surabondance néanmoins très inégalement répartie selon les sujets abordés. Dans le dossier strictement palestinien, par exemple, les auteurs n'ont la plupart du temps prêté attention qu' aux seuls aspects militaires et diplomaticopolitiques. Ils ont ainsi été amenés à ne traiter que de l'Organisation de Libération de la Palestine (OLP), réduite le plus souvent à sa structure de l'Extérieur (Amman, puis Beyrouth et Tunis), elle-même prioritairement perçue à travers son discours diplomatique et sa pratique de la violence. La société, de l'Intérieur (Cisjordanie et bande de Gaza) comme de l'Extérieur, s'est ainsi retrouvée marginalisée.

L'approche académique et médiatique mais aussi diplomatique de l'islam palestinien s'insère dans cette distorsion générale de l'historiographie. Ce regard pâtit dans le même temps des biais bien connus de l'appréhension commune de l'islam et de l'islamisme dans le monde d'aujourd'hui. Porté au pinacle dès lors qu'il servait à mobiliser la «défense du monde libre» face à la menace soviétique sur les rives sud de l'ex-URss, passé sous silence mais discrètement aidé dès lors qu'il sert tout simplement les intérêts occidentaux locaux ou régionaux, il se trouve désigné à la vindicte populaire comme terroriste et/ou rétrograde dès lors qu'il s'oppose à ces mêmes intérêts ou manifeste de la réticence à les servir.

Devenu fer de lance de la lutte anti-israélienne à la fin des années 1980, l'islamisme palestinien s'est ainsi retrouvé communément caractérisé en simples termes de terrorisme et immédiatement disqualifié, la société porteuse de son projet étant une fois encore effacée. Élevée au rang de garant de la paix et de l'équilibre régional après avoir été exécrée durant des décennies, l'Olp et l'Autorité Palestinienne (AP), son surgeon en charge de l'autonomie, sont dorénavant couramment offertes à la perception comme le dernier rempart «laïque et moderne» dressé contre la «barbarie islamiste».

Loin de cette vision, le texte normatif de la Palestine en construction, à travers ses multiples moutures élaborées entre 1993 et 2000, témoigne des évolutions considérables connues par les Palestiniens (et les peuples alentour) ces trois dernières décennies. Ainsi, si l'OlP réclamait 
dans les années 1970 l'édification d'un «État démocratique et non confessionnel» en Palestine, aujourd'hui le Conseil Législatif palestinien d'autonomie (CLP) fait de l'islam «la religion officielle» et de la sharia islamique «une source principale de la législation» de l'État en construction tandis que la toute récente commission formée par le Conseil Central de l'OLP (CCOLP), instance intermédiaire entre le Comité Exécutif (CEOLP) et le Conseil National palestinien (CNP, Parlement en exil), en fait «la source principale de la législation». C'est ainsi sous l'angle du rôle accordé à la religion que nous aborderons les différents projets constitutionnels palestiniens sans pouvoir entrer dans le détail de l'ensemble de leurs dispositifs institutionnels.

\section{CONSTITUTION ET LOI FONDAMENTALE : CHRONOLOGIE DES VERSIONS}

Proclamant l'État de Palestine le 15 novembre 1988, le CNP avait logiquement adopté concomitamment une résolution appelant à la rédaction d'une constitution. Yasser Arafat, président du CEOLP, s'était cependant opposé à l'époque à ce que cette charge soit déléguée au CCOLP pour en accélérer l'exécution. Plusieurs drafts auraient alors été rédigés par le Comité Juridique du CNP présidé par Anîs Al-Qâsim, un juriste qui s'était fait connaître vingt ans plus tôt en rédigeant la Charte Nationale palestinienne, avec le concours d'Eugène Cotran, autre juriste palestinien exerçant des fonctions de Crown Court Judge en Grande-Bretagne. Aucun de ces drafts ne retint néanmoins l'intérêt du CEOLP, lequel ne présenta jamais le texte au CCOLP (QASEM (AL-), A. 1992-1994: 188 et 196-197).

En novembre 1993, tandis que l'OLP se préparait à prendre en charge Gaza et Jéricho suite à la signature des accords d'Oslo, le CEOlP établissait un Haut Comité Juridique chargé de la même tâche constituante. Le président du Comité Juridique du CNP, le même Anîs Al-Qâsim, en fut nommé responsable en dépit de ses réticences à forger un texte constitutionnel pour une simple expérience d'autonomie dont l'efficacité restait encore à démontrer. Quatre versions successives d'un document qui visait à mettre en place le cadre institutionnel d'une démocratie représentative, peuvent lui être imputées.

Un premier texte, qualifié de «projet de constitution (dustûr) de l'Autorité palestinienne pour la période intérimaire», est examiné dès le 5 décembre 1993 par le CEOlP qui décide de le faire circuler largement pour mobiliser le plus d'experts et de points de vue possible ${ }^{1}$. Bien que basé sur les projets de textes antérieurs, cette version est qualifiée de «first draft» par Anîs Al-Qâsim lui-même.

Le Comité Juridique du CNP, réuni à Amman le 10 décembre suivant en présence du speaker par intérim, du secrétaire du CEOLP, et de plusieurs éminents juristes palestiniens, amende ce premier texte; prenant également en considération les nombreuses remarques émises soit par des particuliers soit par des institutions, organisations de femmes et Comité Judiciaire du CNP, le Comité Juridique publie une nouvelle version, la deuxième de la série, qualifiée de «projet de statut fondamental (al-nidhâm al-asâsî) de l'Autorité nationale palestinienne pour la période intérimaire» ${ }^{2}$.

Le 4 février 1994, cette deuxième mouture fait à son tour l'objet d'un vaste débat populaire, cette fois-ci dans le cadre d'une conférence organisée à Jérusalem par le Jerusalem Media and Communication Center (JMCC), agence de presse et think-tank proche du Parti du Peuple Palestinien (ex-communiste) (PPP). L'intégration des observations, qui portaient principalement sur la question des pouvoirs du président, débouche à son tour sur une troisième mouture, qui se trouve qualifiée cette fois de "projet de statut constitutionnel (al-nidhâm al-dustûrî) de l'Autorité nationale pour la période intérimaire». Elle est publiée le $1^{\text {er }}$ mai $1994^{3}$. 
De nouveaux débats publics sont alors organisés, à Naplouse par exemple le 8 décembre 1994 à l'initiative du Center for Palestine Research and Studies (CPRs), autre think-tank mais cette fois plutôt proche de Fath. Alors présidée par Hanân Achrâwî, l'ancienne porte-parole de la délégation palestinienne à Washington, la Palestinian Independent Commission for Citizen's Rights (PICCR), récemment créée par Yasser Arafat comme ombudsman de la nouvelle Autorité et chargée de travailler à intégrer les droits de l'homme dans la législation palestinienne, propose également un certain nombre d'amendements (http://www.piccr.org/ et FrIEDMAN, G., 1999). Une réunion est tenue à cette fin en juillet 1995 à laquelle participait également le chef du département de la fatwa et de la législation du ministère palestinien de la Justice. Entre temps, la signature des accords d'Oslo II avec leurs dispositions sur l'Autorité exécutive et le Conseil d'autonomie avait conduit à revoir profondément le texte. Une quatrième version a donc été mise en chantier, une nouvelle fois sous la direction d'Anîs Al-Qâsim. Tenant compte des remarques faites lors des diverses consultations avec un renforcement des dispositifs visant à la protection des droits de l'homme et intégrant les nouvelles réalités issues des accords, elle a officiellement été présentée le 11 décembre 1995 au ministère de la Justice de l'Ap sous le titre de «projet de statut constitutionnel (al-nidhâm al-dustûrî) pour la période intérimaire»"

Ce quatrième draft constitue l'ultime version élaborée sous la direction d'Anîs Al-Qâsim et les projets ultérieurs sont l'œuvre d'institutions académiques de Cisjordanie, du ministère palestinien de la Justice, du Comité Juridique du CLP ou encore d'un énième Comité créé par l'exécutif. Seuls les projets rédigés par le CLP, cependant, ont in fine bénéficié du caractère officiel consacré par un vote.

Les élections du Conseil d'autonomie et du président de l'Autorité exécutive s'étant tenues le 20 janvier 1996 (Legrain, J-F., 1999), il incombait au CLP d'adopter une loi fondamentale conformément à l'article 3-7 du second accord intérimaire sur les modalités de l'autonomie palestinienne (dit «de Taba» ou «Oslo II») signé à Washington le 28 septembre 1995. Élu président de l'Autorité, Yasser Arafat dès le 3 février chargeait officiellement de préparer le projet de «constitution provisoire» (al-dustûr al-mu'aqqat) une commission faite de politiques et de juristes. Anîs Al-Qâsim y participait en compagnie de Salîm Zanûn, speaker du CNP, de Nabîl Chath, élu du Clp et ministre de la Planification et de la Coopération internationale, de Frayh Abû Middayn, également élu du Clp et ministre de la Justice, et du juriste Eugène Cotran, déjà consulté antérieurement. De façon intentionnelle (tant Yasser Arafat a manifesté de distance pour ce travail constitutionnel) ou non, cette commission n'a jamais bénéficié d'un mandat clair ni d'un cadre précis concernant tant les méthodes d'examen du projet que ses propres relations avec le CLP et le comité juridique du CNP. Les résultats de son travail, si travail il y a eu, n'ont jamais été rendus public.

Entre temps, toujours au printemps 1996, une cinquième version est élaborée sur la base des premiers projets mais cette fois sous la direction du Centre de droit de l'université de Bir Zeit et d'Al-Haq, une organisation déjà ancienne de défense des droits de l'homme située à Ramallah $^{5}$. Les modifications apportées mettent l'accent sur la préservation des droits de l'homme. Le nouveau texte, qui reprend pour titre celui de «projet de loi fondamentale (alqânûn al-asâsî) palestinienne pour la période intérimaire», encadre également plus fortement l'Autorité exécutive, limitant ses pouvoirs et les soumettant à de nombreux engagements internationaux.

Dès sa deuxième session début avril 1996, le Conseil a pour sa part demandé à l'Autorité exécutive pour en débattre communication du projet de «constitution provisoire» commandé 
en février ${ }^{6}$. Début mai, en l'absence de réponse de l'exécutif, le Comité Juridique du Conseil se voyait chargé par le Conseil de travailler à un projet de «constitution» qui lui serait ensuite soumis.

Présidé parl'avocat Abd Al-Karîm Abû Salâh, élu «indépendant» de Gaza mais appartenant en réalité au courant religieux de Fath, le Comité Juridique comptait alors treize membres dont quatre juristes de profession, Abd Al-Karîm Abû Salâh, Sulaymân Abû Snayna (élu Fath, Hébron), Ziyâd Abû Zayyâd, indépendant mais Fath en réalité, Jérusalem) et Faraj Al-Sarrâf (Fath, Gaza). Si dix d'entre eux relevaient de l'orbe de Fath (investis ou non par le mouvement lors de l'élection), cinq pouvaient néanmoins être qualifiés d'islamistes : Abd Al-Karîm Abû Salâh (indépendant, Gaza), Sulaymân Abû Snayna et Rafî̀ Al-Natcha (Fath, Hébron) relèvent de l'aile religieuse de Fath; Imâd Al-Fâlûjî (Fath, Gaza-nord) est un transfuge de Hamas; Sulaymân AI-Rûmî (indépendant, Rafah) est un imâm indépendant, mukhtâr d'une famille bédouine et proche du courant piétiste tablîgh. Un seul seulement appartenait à une organisation d'opposition, le FPLP, Râ'fât Al-Najjâr (indépendant, Khân Yûnis) tandis que participait également au Comité l'inclassable Abd Al-Jawâd Sâlih (indépendant, Ramallah), éphémère ministre de l'Agriculture. Une seule femme siégeait, Jamîla Sîdum (Fath, Dayr Al-Balah) et un chrétien, l'avocat Faraj Al-Sarrâf. Trois étaient des «returnees» ces cadres de l'Olp revenus de Tunis avec l'Ap, Jamîla Sîdum, Rafîq Al-Natcha et Azzâm Al-Ahmad (Fath, Jénine), et sept réfugiés, Jamîla Sîdum, Sulaymân Al-Rûmî, Abd Al-Azîz Châhîn (Fath, Rafah),Imâd AlFâlụ̂î̀, Farah Al-Sarrâf et Muhammad Al-Hawrânî(Fath, Hébron). Les élus de Gaza, au nombre de sept, l'emportaient ainsi sur ceux de Cisjordanie.

En dépit des difficultés liées au blocus israélien des territoires ${ }^{7}$, le Comité Juridique du CLP parvient à accomplir son travail, soit, mais de façon improbable, à partir d'une version fournie directement par la commission créée en novembre 1993, soit plutôt à partir du quatrième draft d'Anîs Al-Qâsim. Son projet, le sixième, est achevé à la mi-juin 1996 et à nouveau offert au débat à travers la presse comme «projet de statut constitutionnel provisoire (al-nidhâm aldustûrî al-mu'aqqat) de l'Autorité nationale palestinienne pour la période intérimaire» ${ }^{8}$.

Parallèlement à ces efforts, l'exécutif, via la direction de la fatwa et de la législation du ministère de la Justice, travaillait sur un projet qui lui était propre. Septième de la série, il est rendu public sous le titre de «projet de statut fondamental (al-nidhâm al-asâsî) de l'Autorité nationale palestinienne»" . Il n'a jamais, semble-t-il, fait l'objet d'un examen direct et officiel de la part du CLP10 .

A pareille époque, l'université Najâh de Naplouse (draft 8) élaborait également son propre projet de même que Ali Khashan, dean de la Jerusalem's Palestinian School of Law de l'université al-Quds, qui rédige une «loi fondamentale (al-qânûn al-asâsî) de l'État de Palestine» $(\text { draft } 9)^{11}$.

Poursuivant son travail, le Comité Juridique du CLP publiait le 9 juillet 1996 une nouvelle version amendée, dorénavant porteuse du chiffre 10, et la remettait à la fois au Président de l'AP et au $\mathrm{CLP}^{12}$. De «constitution» (al-dustûr), le texte devenait «loi fondamentale» (al-qânûn alasâsî̀). Le CLP chargeait parallèlement sa présidence, le Conseil des ministres et ses Comités Juridique et politique de rédiger un préambule à cette loi. Un comité, constitué de huit membres et tous élus du CLP, est alors investi de cette tâche. Tandis que la parité géographique est respectée entre Gaza (Nâhid Al-Rayyis élu de Gaza-ville, Frayh Abû Middayn et Sadî AlKrunz de Gaza-centre ainsi que Hasan Asfûr de Khân Yûnis) et la Cisjordanie (Hanân Achrâwî de Jérusalem, Azmî Al-Chuaybî de Ramallah ainsi que Muhammad Al-Hawrânî et Sulaymân 
Abû Snayna d'Hébron), l'éventail politique est quant à lui quasi monochrome, sept ont en effet été élus sur les listes Fath et la huitième, élue indépendante, est une proche du mouvement. Ancien cadre du PPP ex-communiste, Hasan Asfûr est maintenant totalement intégré à l'AP tandis que Azmî Al-Chuaybî est l'un des dirigeants de Fida, scission du Folp datant de 1993 qui a bien du mal dorénavant à se distinguer de Fath. Une fois encore, les religieux marquent le comité de leur présence : Nâhid Al-Rayyis, ancien adjoint d'Abû Jihâd, a appartenu dans les années 1980 à une cellule islamiste de Fath; Sulaymân Abû Snayna, comme déjà souligné, appartient lui aussi à l'aile religieuse de Fath ainsi que Sadî Al-Krunz, par ailleurs viceprésident d'un Mouvement Palestinien de la Paix et de l'Entente (Harakat Al-Salâm wa-lTafâhum Al-Filastîniyya) fondé en 1993 et qui, ardent défenseur des accords d'Oslo, se donne pour but de «rechercher des traits d'union entre les religions islamique et juive» (Legrain, J-F., 1996). C'est au tour, cette fois-ci, de Hanân Achrâwî d'assurer une présence chrétienne.

Le 17 juillet 1996, lors d'une séance orageuse du CLP, Yasser Arafat manifestait une nouvelle fois ses réticences devant tout travail constitutionnel et décidait de retirer le texte du débat : «Ce projet, déclarait-il, n'aurait pas dû vous parvenir avant d'avoir été revu par le comité ad hoc du CNP. C'était une erreur. Nous ne devons pas contourner l'autorité de l'OLP». Un compromis est néanmoins trouvé entre Yasser Arafat et le CLP au terme duquel ce dernier pourrait poursuivre sa discussion sur le projet de loi tandis qu'un débat parallèle serait mené au sein de l'Olp. De nouvelles esclandres surgissent lors des séances du 23 et du 30 juillet conduisant Yasser Arafat à quitter la salle du Conseil et Ahmad Qray, le speaker, à démissionner quelques heures de sa fonction.

Suite à ses délibérations, le CLP adopte le 11 octobre 1996 en première lecture son propre texte dorénavant doté d'un préambule, le onzième de la série, et le soumet à l'exécutif ${ }^{13}$. Faute de réponse de sa part, début juillet 1997 soit presque un an plus tard le CLP décide de reprendre l'examen de son texte. Amendé, le texte (le $12^{\mathrm{e}}$ ) est adopté en deuxième lecture le 17 septembre 1997. En l'absence, une fois de plus, de réaction de la part de l'exécutif, le texte $\left(13^{\mathrm{e}}\right)$ est définitivement adopté en troisième lecture le 2 octobre 1997 à l'unanimité moins une abstention $^{14}$.

Trois ans après que le Conseil a achevé les trois lectures nécessaires à l'adoption de toute loi, en dépit de la nécessité juridique selon laquelle la loi fondamentale devait être adoptée avant la fin de la première saison du Conseil (soit le 4 août 1996), et en dépit des assurances données par Yasser Arafat lui-même d'une prochaine promulgation du texte en attente de sa signature, aucune loi fondamentale n'était encore entrée en vigueur.

Entre temps, le CCOLp, réuni à Gaza les 27 et 28 avril 1999, chargeait le CeOlp et la présidence du CNP d'accélérer la marche vers la souveraineté à travers la formation de diverses instances parmi lesquelles figurait une «Commission de la Constitution de Palestine». Aucune référence n'était faite au travail déjà accompli. Appelée à travailler dans le cadre d'un Haut Comité de la Ligue des États arabes lui-même présidé par le secrétaire général de la Ligue, Ismat Abd al-Magîd, la délégation palestinienne était menée par Nabîl Chath, ministre de la Planification et de la Coopération internationale. Une seule réunion, semble-t-il, s'est tenue au Caire (le 7 juin 1999), l'ensemble du travail ayant ensuite été mené en Palestine même dans le cadre d'une commission baptisée «Commission de rédaction (lajnat al-siyâgha) de la Constitution» dont le lien avec la Ligue demeure obscur. Animée par Ahmad Mubârak AlKhâlidî, professeur de droit constitutionnel à l'université Najâh de Naplouse, elle comprenait sept autres membres ${ }^{15}$. L'avocat Abd Al-Karîm Abû Salâh, président du Comité Juridique du 
CLP qui avait déjà participé à ce titre à la rédaction du texte adopté par le Conseil et Khayrî Hasan, président de la commission juridique du CcOlp, faisait partie du groupe au côté de Ali Khashan, dean de la Jerusalem's Palestinian School of Law et déjà auteur d'un texte constitutionnel propre, de Nâfi al-Hasan, professeur de droit humanitaire dans la même école de l'université al-Quds, et de Kamâl al-Astal, professeur de science politique à l'université alAzhar de Gaza. Mahmûd al-Ajramî, fonctionnaire de l'Ap et porte-parole du Front Démocratique de libération de la Palestine (FDLP de Nayef Hawatmeh) à Gaza, assurait la participation de l'opposition égale (également marquée avec celle de Nâfi al-Hasan du même FolP) tandis que la présence de Camille Mansour pouvait être lue à la fois à la lumière de sa fonction, directeur du Centre de droit de l'université de Bir Zeit, mais aussi de sa confession, chrétienne ${ }^{16}$. A la différence des précédentes commissions, les juristes étaient majoritaires mais également les returnees (Nâfi al-Hasan, Mahmûd al-Ajramî, Khayrî Hasan et Camille Mansour), tous étant réfugiés de 1948 comme Ali Khashan.

Le 4 juillet 2000, la presse palestinienne publiait les grandes lignes de son «projet de constitution provisoire (al-dustûral-wuwaqqat)» $\left(\mathrm{le} 14^{\mathrm{e}}\right)^{17}$, une deuxième version étant remise à l'exécutif le 8 juillet (soit le $15^{\mathrm{e}}$ ) $^{18}$. Élaboré cette fois loin de toute consultation populaire et objet, semble-t-il, de la vindicte du ministère de la Justice, le texte était entièrement nouveau et rompait ainsi avec les treize premières versions de la série ${ }^{19}$. Ultime témoin des errements de l'Olp et de l'Ap en la matière, dans un geste qui aurait voulu réconcilier ministère de la Justice et ministère du Plan et de la Coopération internationale, la présidence du CNp créait le 15 août 2000 une nouvelle commission, sous le nom cette fois de «Commission de proclamation (ilân) de la constitution». Placée sous les auspice du speaker du CNP, Salîm Zanûn, et la présidence de Jamâl al-Sûrânî, président de la commission juridique du CNP, cette énième commission ne compte parmi ses huit autres membres qu'une seule personnalité ayant participé aux travaux antérieurs, Nâfi al-Hassan. Tous exercent des fonctions juridiques académiques (Nâfi alHassan à l'université al-Quds, Rafî̀ Abû cAyyâch, professeur de droit constitutionnel à l'université al-Quds; Haytham al-Zubî, de Bir Zeit; Fathî al-Wuhaydî, professeur de droit constitutionnel à al-Azhar de Gaza), privées (Abd al-Rahmân Abû al-Nasr, président de l' Ordre des avocats) ou administratives (Hannâ Isâ Haylâna, directeur général au ministère de la Justice, chrétien; Isâ Abû Charâr, juge de la Cour d'Appel de Ramallah et ancien officier de l'armée jordanienne chargé des affaires juridiques), le rapporteur de la commission étant Salâh Abû Jazar. Deux mois plus tard, cette commission ne se serait encore jamais réunie tandis que le CCOLP réuni à Gaza les 9 et 10 septembre 2000 renouvelait son mandat accordé au CEOLP, à la présidence du CNP et au CLP de finaliser la déclaration constitutionnelle. Le 15 septembre, Jamâl Al-Sûrânî annonce que le comité a rédigé son projet (le $16^{\mathrm{c}}$ de la série $)^{20}$.

\section{LES ÉVOLUTIONS DU TEXTE À TRAVERS SES VERSIONS SUCCESSIVES}

En dépit de leur forme qui rappelle indéniablement celle de la constitution d'un État, les divers drafts élaborés de 1.993 à 1997 n'étaient censés s'exercer que durant la période intérimaire. Ces textes cependant, rédigés par les plus hautes instances officielles palestiniennes ou même adoptés par vote, peuvent aussi être lus comme donnant une préfiguration du régime de l'État revendiqué.

\section{L'Autorité palestinienne, surgeon de l'OLP}

A l'exception des textes élaborés par Ali Khashan et par la «Commission de rédaction de la constitution» tous ces drafts, sous une forme ou sous une autre, font état d'un lien 
institutionnel entre l'Ap, dont les modes de fonctionnement sont censés être fixés par le texte, et l'OLP. Rédigé avant l'accord de Taba qui jetait les bases juridiques de l'élection de l'Autorité exécutive, le premier $d$ raft témoigne de l'articulation la plus forte entre les deux institutions puisque, selon son article 4, c'est «le président du Comité exécutif [de l'Ol.P] [qui] est le chef suprême de l'Autorité exécutive. Il exercera les prérogatives qui lui sont dévolues dans le statut fondamental (al-nidhâm al-asâsî) de l'Otp, les résolutions du Conseil national et du Conseil central et dans cette loi». C'est donc devant le CeOlp que le Président est appelé à prêter serment (article 5) et c'est ce même CEOLP qui est appelé, selon l' article 13, à désigner les membres d'un «conseil appelé 'gouvernement de l'autorité nationale'(hukûmat al-sulta al-wataniyya)». Ce dispositif était conforme à la Déclaration de principes sur des arrangements intérimaires d'autonomie signée à Washington le 13 septembre 1993 (dit également «accord d'Oslo» où il avait été négocié) qui stipulait dans son article 6 que «le transfert de compétence du gouvernement militaire israélien et de son administration civile» se ferait «aux Palestiniens mandatés pour cette tâche». C'était en réponse à cette demande de «mandat» que le CCOLP, réuni à Tunis en octobre 1993, avait adopté une résolution selon laquelle : «1) Il investit le CEOLP de la formation du Conseil de l'Autorité nationale palestinienne dans le cadre de l'étape intérimaire avec des membres du CEOLP et un certain nombre de [personnalités] de l'Intérieur et de l'Extérieur; 2) Yasser Arafat, président du CEOLP, sera Président du Conseil de l'Autorité nationale palestinienne» (Munazzamat Al-Tahrîr Al-Filastîniyya (OLP), Sd., 72). Tandis que les accords israélo-palestiniens parlaient de «l'Autorité exécutive du Conseil d'autonomie», le draft de décembre 1993, à l'instar du CCOLP, décide de caractériser la nouvelle instance en termes de «Conseil» et de «gouvernement de l'Autorité nationale» (al-sulta al-wataniyya), affichant ainsi une supposée adéquation avec l'Autorité appelée par le douzième $\mathrm{C}_{N P}$ tenu au Caire en juin 1974 à être créée sur «toute partie libérée» de la Palestine (Naufal, M. 1995).

Responsables devant le président de l'Ap selon le premier draft (article 18), les ministres deviennent responsables devant le CEOLP avec les deuxième (article 20) et troizième (article 63) drafts. A partir du quatrième draft, du fait de l'élection au suffrage universel du Conseil de l'AP et de son président, les ministres deviennent responsables devant le président et le Conseil (article 83 du quatrième draft, 74 puis 68 dans les versions adoptées par le CLP, mais devant le seul président selon l'article 115 de Ali Khashan).

La même préoccupation visant à articuler l'AP et l'OLP se manifeste dans d'autres articles des drafts 2 et 3. L'article 6 du deuxième draft, par exemple, ajoute parmi les références de l'action du Président les décisions prises par le CEOLP. Son article 9 (devenu 53 dans le $3^{\text {e)}}$, absent du premier draft, stipule par ailleurs qu'en cas de décès du Président, c'est au speaker du CNP d'assurer son intérim durant 60 jours dans l'attente d'une nouvelle élection) ${ }^{21}$. Le troisième draft (articles 50,51 et 58), pour sa part, stipule que le serment demandé au Président se fera devant le bureau de la présidence du CNP.

Rédigés après la tenue des élections du Conseil et du Président de l'Autorité exécutive, le quatrième draft et les versions ultérieures témoignent du sevrage institutionnel de l'Ap vis-àvis de l'OLP. Le préambule ajouté par le CLP, dans un discours quelque peu grandiloquent, se charge néanmoins de rappeler que «la naissance de l'Autorité nationale palestinienne sur le sol de la patrie, la Palestine, la terre des pères et des ancêtres, s'insère dans la dure lutte ininterrompue du peuple palestinien qui a offert parmi ses fils des milliers de martyrs, de blessés et de prisonniers, pour parvenir à la réalisation de ses droits nationaux inaliénables que sont le droit au retour, à l'autodétermination et à l'édification de l'État palestinien indépendant avec Jérusalem pour capitale sous le leadership de l'OLP, unique représentant légitime du peuple 
arabe palestinien où qu'il soit». Ancien, ce lien historique se maintient à travers des dispositifs institutionnels croisés. Élu au suffrage universel par les Palestiniens de Cisjordanie et de la bande de Gaza, c'est encore devant le bureau de la présidence du CNP que l'article 69 du quatrième draft impose au Président de l'Autorité exécutive de prêter serment tandis que l'article 117 souligne que la loi fondamentale «n'affecte en rien les prérogatives et les pouvoirs dévolus à l'Olp et à ses institutions et parmi eux sa [capacité à] représenter le peuple palestinien dans les relations internationales [...]». Les versions adoptées par le CLP maintiennent le lien en exigeant du Président élu de prêter serment «devant le Conseil législatif et en présence du speaker du CNP et du Président de la Haute Cour» (article 57 devenu ensuite 52). Le texte de la «Commission de rédaction de la constitution», en revanche, reste curieusement discret sur l'Olp. Ignorée du préambule, elle n'est mentionnée que dans le chapitre dédié au pouvoir législatif, ses lois et règlements faisant partie de l'héritage juridique palestinien.

Alors que les versions antérieures, lorsqu'elles le mentionnaient, de même que le texte de Ali Khashan (article 6) et celui de la «Commission de rédaction de la constitution» (article 8) donnaient une description détaillée du drapeau palestinien, les versions adoptées par le CLP (article 8) s'en abstiennent mais soulignent la continuité avec l'OLP : «Le drapeau de Palestine, avec ses quatre couleurs et les dimensions de celui adopté par l'OLP, est le drapeau officiel du pays».

\section{Une démocratie représentative}

L'ensemble des versions disponibles mettent en place un cadre institutionnel typique d'une démocratie représentative même si les insistances ou garanties varient d'une version à l'autre. Toutes les versions officielles antérieures à 1997, par exemple, stipulent que «le peuple palestinien est la source des pouvoirs qu'il exerce durant la période intérimaire à travers les pouvoirs législatif, exécutif et judiciaire selon les dispositions de ce statut» (article 1 ou 2 selon les versions), le ClP tenant à préciser dans ses versions que cet exercice se fait «sur la base du principe de la séparation des pouvoirs", principe ensuite décliné dans le texte même de la loi. La version du ministère de la Justice pour sa part utilise d'autres termes en affirmant dans son article 2 que «c'est le peuple palestinien qui est détenteur de la souveraineté qu'il exerce de façon détaillée dans ce statut», son article 50 stipulant la séparation des pouvoirs.

Les versions 2 à 4 stipulent également dans leur article 2 que «le régime en Palestine sera un régime démocratique et parlementaire basé sur le pluralisme des partis, la prise en compte par la majorité des droits et des intérêts de la minorité et le respect par la minorité des décisions de la majorité», expression encore présente dans la version du ministère de la Justice (article 3 ) et également présente en des termes comparables dans l'article 12 de la "Commission de rédaction de la constitution» mais remplacée dans les versions adoptées par le CLP par l'affirmation (devenue article 5) selon laquelle " le régime en Palestine sera un régime démocratique et parlementaire basé sur le pluralisme politique et des partis dans lequel le président de l'Autorité nationale est élu au suffrage direct par le peuple et dans lequel le gouvernement est responsable devant le Président et le CLP».

Cette préoccupation peut se résumer dans le principe plusieurs fois martelé : «La souveraineté de la loi est une base du régime en Palestine» [souligné par moi] (article 82 et tête de chapitre dans le premier $d$ raft, devenu 89 et tête de chapitre dans le deuxième puis article 36 et tête de chapitre dans les versions 3 et 4 , et article 42 de la version du ministère de la Justice), principe renforcé dans l'article 6 des versions adoptées par le CLP sous le mode : «Le principe de la souveraineté de la loi est la base du gouvernement en Palestine» [souligné par moi]. L'ensemble 
des versions, en des termes divers plus ou moins détaillés le maximum étant atteint avec le texte de Bir Zeit, font également référence à la reconnaissance de la validité des principales conventions internationales de respect des droits de l'homme.

Dans cette logique selon laquelle «le peuple palestinien est la source des pouvoirs», le premier draft, dans son article 94, précise que «les lois sont promulguées au nom du peuple palestinien», un article reproduit ensuite à l'identique (article 102 du deuxième draft, $104 \mathrm{du}$ $3^{e}, 118$ du quatrième, 10 du chapitre 6 de la version de Bir Zeit, 49 de la version du ministère de la Justice, 108 puis 107 des versions adoptées par le CLP). Dans le même état d'esprit, l'article 99 du deuxième draft (repris à l'identique dans l'article 46 des troisième et quatrième, 94 puis 88 des versions adoptées par le CLP) stipule que «les jugements sont prononcés et exécutés au nom du peuple». L'article 161 de la «Commission de rédaction de la constitution» mentionne également que «les jugements sont émis en conformité avec la constitution et sont promulgués et exécutés au nom du peuple». Avec les versions rédigées par le Comité Juridique du CLP, pourtant, comme dans le cas du texte de la «Commission de rédaction de la constitution», et sans que la contradiction ne semble être apparue aux rédacteurs, la basmalla (la formule coranique «Au nom de Dieu, le miséricordieux, celui qui fait miséricorde») figure en tête de chacun des projets, une islamisation observée par ailleurs dans l'évolution des dispositifs prévus par la $\operatorname{loi}^{22}$.

Le texte élaboré par Ali Khasan tente d'échapper ce type d'ambiguiités. Précédé de la basmalla, à aucun moment il ne fait explicitement du peuple la source des pouvoirs pas plus qu'il ne stipule que les lois sont promulguées au nom du peuple. Si cependant son article 2 précise bien que «le régime en Palestine est démocratique» et que l'article 51 affirme la séparation des pouvoirs, l'article 35 ne fait de la souveraineté de la loi qu' »une source principale du gouvernement et de la société» [souligné par moi], l'article 2 se contentant d'affirmer que «le peuple exerce les manifestations de la souveraineté (madhâhir al-siyâda) [dans le cadre du régime démocratique de Palestine]». L'article 57 de la «Commission de rédaction de la constitution», en revanche, stipule explicitement que «le peuple palestinien est la source des pouvoirs» mais ne fait de la souveraineté de la loi qu'»une source principale du gouvernement» (article 15).

\section{La souveraineté et ses domaines de traduction}

Dans le domaine de la géographie de l'exercice de la souveraineté du peuple, aucune des versions ne précise les frontières de la Palestine. La version élaborée par Bir Zeit et al-Haq mentionne néanmoins que «les gouvernorats de Cisjordanie et de la bande de Gaza constituent une seule unité régionale. Il revient aux autorités palestiniennes de travailler au renforcement de leur unité, de leur intégrité et de leur stabilité» (article 2 du chapitre 8) suggérant ainsi une reconnaissance de facto de l'existence d'Israël entre les deux régions. Seul le texte de la «Commission de rédaction de la constitution» souligne que «la Palestine est la propriété de [toutes] les générations du peuple arabe palestinien [...]» (article 3). Si son article 11 stipule que «la souveraineté sur la Palestine appartient à [toutes] les générations palestiniennes. Elle ne [saurait] disparâtre par obsolescence et nul ne peut l'abandonner», le préambule mentionnait néanmoins l'attente des Palestiniens depuis la résolution 181 du partage à voir s'épanouir le rôle civilisationnel de l'État de Palestine. Ce texte est aussi le seul à souligner que «le droit du Palestinien au retour à son village et à son lieu de naissance est un droit naturel» (article 30).

Concernant Jérusalem, tandis que le premier et le deuxième drafts l'ignorent en tant que capitale (mais la mentionnent à l'occasion des articles traitant des Lieux-Saints, de la liberté 
d'accès et de culte) l'ensemble des versions ultérieures (article 5 des drafts du Comité Juridique du CNP devenu article 3 dans les versions adoptées par le CLP) stipulent que «Jérusalem est la capitale de la Palestine», l'article 5 des versions de Bir Zeit, de Ali Khashan et du ministère de la Justice précisant en outre que «durant la période intérimaire, l'Ap pourra établir son siège n'importe où ailleurs en Palestine». Pareille mention est faite par l'article 66 de Ali Khashan pour le siège du Conseil législatif. L'article 7 du texte de la «Commission de rédaction de la constitution» mentionne pour sa part que «Jérusalem est la capitale de l'État de Palestine». La ville est également le siège du Parlement mais ses sessions peuvent être tenues ailleurs (article 62). Les quatre premières versions stipulent par ailleurs que «la souveraineté sur les richesses naturelles en Palestine est entre les mains du peuple palestinien [...]» (article 93 du premier draft, 101 du $2^{\mathrm{e}}, 6$ du troisième et du quatrième, et 6 de la version de Bir Zeit); reprise en termes comparables dans le texte du ministère de la Justice (article 8) et dans celui de la «Commission de rédaction de la constitution» (article 18), la notion est ignorée des textes adoptés par le CLP.

La souveraineté du peuple palestinien passe également par l'adoption de l'arabe comme langue officielle. Sa mention apparaît dès le premier draft et se retrouve dans l'ensemble des versions ultérieures mais dans des dispositifs juridiques assez différents. La première version fait ainsi preuve à la fois de précision et de grande ouverture : selon son article 92 , en effet, «la langue arabe est la langue officielle de Palestine. Il sera possible par une loi d'adopter une autre langue officielle. Ceci n'implique pas la non utilisation d'une autre langue dans les cas précisés par la loi». L'article 3 du deuxième draft reprend le même texte mais supprime la possibilité légale d'adopter une autre langue officielle, possibilité qui ne sera donnée par aucune autre version ultérieure. A ce dispositif ouvert, les troisième et quatrième drafts ont préféré la simple affirmation selon laquelle «la langue de Palestine est la langue arabe» (article 3). Les versions adoptées par le CLP reviendront ensuite à la notion d'origine sans toutefois en reprendre les mêmes ouvertures : «La langue arabe est la langue officielle», principe également mentionné dans l'article 3 de Ali Khashan et 4 de la «Commission de rédaction de la Constitution».

Curieusement, à l'exception de la mention de la langue, l'appartenance de la Palestine à l'ensemble arabe est oubliée des premiers drafts et n' intervient (en concomitance avec l'islam) qu'à partir de la première des versions élaborées par le Comité Juridique du CLP à la mi-juin 1996. Son article 2 (dans la version 6 devenu 1 ensuite), en effet, mentionne que «la Palestine fait partie de la grande patrie arabe, le peuple arabe palestinien fait partie de la nation arabe et l'unité arabe constitue un objectif que le peuple palestinien cherche à atteindre» ${ }^{23}$. La version du ministère de la Justice stipule pour sa part que «le peuple palestinien fait partie de la nation arabe et sa terre fait partie de la patrie arabe». Le préambule des versions adoptées par le CLP parle quant à lui de «peuple arabe palestinien». Ali Khashan insiste également sur l'arabité : «La Palestine est un État [ajouté à la main] arabe indépendant et souverain. Son peuple fait partie de la nation arabe et cherche à réaliser son unité totale. Région, elle fait partie de la grande patrie arabe. Nul ne peut renoncer à la souveraineté sur elle ni à une de ses parts» (article 7) et l'article 27 parle de «nationalité arabe palestinienne». Le texte de la «Commission de rédaction de la constitution» s'insère dans cette même veine. «La Palestine fait partie de la patrie arabe et de la nation arabe» affirme son article 2 et la qualification de «peuple arabe palestinien» est récurrente tout au long du texte.

\section{L'islam comme élément de souveraineté}

Les quatre premiers drafts s'étaient abstenus d'aborder les questions de la religion de l'État et des sources de la législation. Il ne s'agissait en rien, selon Anîs Al-Qâsim, d'un oubli mais 
de la volonté de reporter une prise de décision susceptible de diviser à la rédaction d'une constitution définitive élaborée en toute liberté dans le cadre d'un État souverain (Qasem (Al), A. 1992-1994, 199-200).

La religion n'en était pas pour autant ignorée et figurait dans divers dispositifs concernant les libertés. Ainsi, le premier draft, dans son article 62, garantit que «toute personne jouira de la liberté de pensée, de conscience et de religion» (1'article 67 de la deuxième mouture supprime la mention de la liberté de religion et la remplace par celle de la liberté d'expression, correction reprise par l'article 14 de la troisième mouture, ou 29 du ministère de la Justice), une affirmation complétée par l'article 79 (article 18 des versions adoptées par le CLP), selon lequel «la liberté de foi $(a q \hat{\imath} d a)$, et de pratique des rites religieux est garantie sous réserve de l'absence de trouble de l'ordre public ou des bonnes mœurs» (l'article 34 de la version du ministère de la Justice mentionne la même garantie en omettant toute limite), et par l'article 80 qui stipule que «l'accès aux Lieux-Saints, aux bâtiments et aux lieux [sic, le texte ici utilisé ayant sans doute oublié l'adjectif «religieux» présent dans le deuxième draft] avec l'intention de les visiter est garanti pour l'ensemble des citoyens et des étrangers sans distinction, de même [qu'est garantie] pour les fidèles la liberté d'y pratiquer le culte dans le cadre du respect de la politique de sécurité, de l'ordre public ou des bonnes mœurs» (repris dans l'article 36 de la version du ministère de la Justice).

Dans le cadre du principe de la souveraineté de la loi, l'article 83 (repris en des termes comparables dans l'article 91 du deuxième draft, 38 des troisième et quatrième, neuf des versions adoptées par le CLP) stipule également que «les gens, quels qu'ils soient, sont égaux devant le [système] judiciaire. Tous, quels qu'ils soient, sont égaux devant la loi et bénéficient sans aucune discrimination d'un droit égal de protection, que cette discrimination soit due à la race, à la couleur, au sexe, à la langue, à la religion, à l'opinion politique, à l'origine, [...]».

Quoique vague dans sa définition des bénéficiaires, l'article 73-2 stipule enfin que «les minorités (al-aqalliyât) et autres [sic, la maladresse de la phrase tient peut-être à l'oubli d'un mot dans la source utilisée, la deuxième mouture mentionnant dans son article 78-2 au contenu identique «les minorités religieuses et autres», texte repris à l'identique dans l'article 25-2 des troisième et quatrième drafts] auront le droit d'établir des écoles privées [...]». Ce lien entre «minorités religieuses» et écoles privées n'apparaît plus dans les versions adoptées par le CLP qui se contentent dans leur article 24-d de mentionner : «Les écoles et instituts privés suivront les curricula mis en place par l'Autorité nationale [...]» pas plus que dans la version du ministère de la Justice qui autorise «les individus et les organismes» (article18-2) à ouvrir de telles écoles.

Ignorant Jérusalem comme capitale politique comme déjà souligné, le deuxième draft inclut pourtant la ville dans la Palestine dont il trace le cadre constitutionnel mais dans le cadre de la défense de certaines libertés et sous un mode d'appréhension religieux. C'est en effet à l'occasion des articles traitant des Lieux-Saints, de la liberté d'accès et de culte que Jérusalem est mentionnée : «Jérusalem est une ville sainte pour les trois religions célestes. En devoir de fidélité au patrimoine spirituel de la Palestine, les autorités palestiniennes se doivent d'aménager les conditions de la coexistence et de la tolérance entre les religions à Jérusalem et dans le reste de la Palestine» (article 84). Les articles 85 et 86 reprennent ensuite les articles 79 (en ajoutant la liberté de culte à la liberté de foi et de pratique des rites religieux) et 80 du premier draft déjà cités. Les troisième et quatrième drafts avec leurs articles 31 à 33 reproduisent les mêmes dispositifs. La version de Bir Zeit (article 22 du chapitre 2) comme celle du ministère de la 
Justice (article 19) reproduisent ce texte sur Jérusalem, ville sainte, mais les textes adoptés par le CLP l'ignorent comme celui de Ali Khashan. A cette occasion, les auteurs du deuxième draft ont introduit la notion de «patrimoine spirituel» de la Palestine; le préambule des versions adoptées par le CLP parlera de «composantes spirituelles». Si aucun des textes n'en donne une définition précise, la référence dans le même contexte aux «religions célestes» renvoie à un univers explicitement musulman.

Le serment imposé dans des termes identiques depuis le premier draft au Président de l'AP lors de son intronisation et aux membres du Conseil s'insère lui-même dans une vision religieuse du monde en l'absence toutefois de connotation confessionnelle particulière : «Je jure devant Dieu le Très-Grand d'être loyal à la patrie et à ses [principes] sacrés, à son héritage national [...]" (articles 5 et 16 dans le premier draft, devenus 7 dans le deuxième, 51 dans le troisième, 69 dans le quatrième, puis 35 et 57, 36 et 52 dans les versions adoptées par le CLP).

Dans le cadre de la description des rouages de l'Ap, le premier draft prévoyait également dans son article 30 qu'un «bureau de la législation et de la fatwa» sera établi sous la direction d'un conseiller juridique nommé par le Président. De façon générale, ce dîwân aura pour prérogatives de fournir le conseil juridique au Conseil et aux divers départements dans les affaires qui lui seront présentées et de préparer les projets de lois, les motions législatives et les décrets. Administrativement, ce dîwân dépendra du département de la Justice». Cette institution disparaît ensuite du deuxième $d r a f t^{24}$.

L'entrée en force de l'islam intervient en fait à partir de la première des versions élaborées par le Comité Juridique du CLP à la mi-juin 1996 et se retrouve avec quelques modifications dans les versions adoptées ultérieurement par le CLP lui-même, les textes de Ali Khashan et de la «Commission de rédaction de la constitution» témoignant de l'attachement à l'islam le plus poussé. Cette «islamisation» va de pair avec la «redécouverte» de l'appartenance de la Palestine à l'ensemble arabe déjà mentionnée.

C'est à partir de la sixième mouture en effet que l'islam est proclamé «religion officielle en Palestine», expression que l'on retrouve à l'identique dans les versions ultérieures adoptées par le CLP, mais néanmoins dans des contextes différents. La version adoptée en première lecture avait en effet retenu pour article 4-1 : «La Palestine est le berceau des trois religions célestes. L'islam est la religion officielle en Palestine et les autres religions [y jouissent] du respect et de la sanctification requis» ${ }^{25}$. Cette appréhension religieuse de la Palestine disparaît entre la deuxième et la troisième lecture du texte au terme d'un débat. Le texte ultime retient donc pour article 4-1 le texte suivant : «L'islam est la religion officielle en Palestine et les autres religions célestes [y jouissent] du respect et de la sanctification requis».

Cette caractérisation n'entraîne cependant pas d'identification confessionnelle du Président. Aucune des versions consultées ne prescrit d'ailleurs de caractère pré-requis pour une candidature à ce poste, à l'exception de celles du ministère de la Justice qui exige de tout candidat qu'il soit «Palestinien des deux pères, qu'il jouisse de l'ensemble des ses droits civils et politiques et soit âgé de 40 ans au moins» (article 78) et de Ali Khashan pour qui le candidat à la présidence doit être «Arabe palestinien des deux pères et jouir de l'ensemble des ses droits civils et politiques» (article 91).

La mention de la sharia intervient quant à elle à partir du septième draft élaboré par le ministère de la Justice selon lequel «les principes de la sharia islamique constituent une source principale de la législation» [souligné par moi] (article 4), mention qui apparaît conjointement 
à la proclamation de la langue arabe langue officielle de Palestine mais en l'absence de la mention de l'islam comme religion d'État. Le texte de Ali Khashan fait de la sharia «la source principale de la législation" [souligné par moi] (article 4) mais en l'absence également de toute mention de l'islam comme religion officielle, et la dixième mouture aurait également stipulé que «les principes de la sharia constituent la source principale de la législation» [souligné par moi] (Chase, A. 1997, 39). La version adoptée en première lecture par le CLP, suivie par les versions adoptées en deuxième et troisième lectures, revient quant à elle à l'expression retenue par le septième $d r a f t$ ne faisant de la sharia qu' une source principale de la législation, et non la source (article 4-2). Trois ans plus tard, la «Commission de rédaction de la constitution» faisait des «principes de la sharia la source principale de la législation» [souligné par moi] et de l'islam «la religion officielle de l'État».

Conjointement à l'introduction de la sharia comme référence juridique, les moutures les plus récentes enferment explicitement le citoyen dans une appartenance à une communauté religieuse par un certain nombre de dispositifs. La version rédigée par Bir Zeit (article $6 \mathrm{du}$ chapitre 6) comme celle du ministère de la Justice s'étaient contentées de mentionner trois catégories de tribunaux, réguliers, religieux et spéciaux, sans en définir le champ de compétence. L'article 98 de la mouture adoptée en première lecture par le CLP, repris dans l'article 114-b de la version adoptée en troisième lecture, assujettit explicitement les questions de droit personnel à des cours religieuses. L'article 96 du premier draft $\left(105\right.$ du $2^{\mathrm{e}}, 107 \mathrm{du} 3^{\mathrm{e}}, 121$ du quatrième, repris sous une forme légèrement différentes dans les articles 110 puis 109 des versions adoptées par le CLP), qui stipulait que «les lois, les motions, les statuts et décrets en vigueur dans la bande de Gaza et en Cisjordanie avant la promulgation de cette loi le demeureront sous réserve qu'ils ne contredisent aucune de ses dispositions et ce jusqu'à la loi les amende ou les annule», laissait place certes au maintien des cours religieuses sans le spécifier. Dorénavant, outre le fait qu'un individu ne saurait être que musulman ou chrétien (le cas des juifs ne se pose pour le moment que pour les Samaritains), sans que le musulman puisse se convertir à l'une des diverses déclinaisons de la foi chrétienne, il se trouve de facto enfermé dans les inégalités induites par les divers codes religieux en matière de mariage (une musulmane ne peut épouser qu'un musulman) ou de divorce (refusé par les chrétiens uniates, courant chez les musulmans), de témoignage, d'héritage, etc. Du coup, certains pourront considérer que l'article 9 sur l'égalité du citoyen face à la loi se trouve invalidé.

Ayant fait de la sharia la source principale de la législation, le texte de Ali Khashan s'abstient de mentionner ces dispositifs confessionnels et ignore toute référence aux «religions célestes», En des termes comparables à ceux employés par les versions rédigées par le CNP et le Clp il stipule que «la liberté de conscience est absolue. La liberté de croyance (itiqâd) est garantie de même que sont protégés les lieux de culte, la liberté de pratiquer les rites des religions ainsi que les processions et réunions religieuses conformément aux coutumes observées» (article 45) et affirme que «les Palestiniens sont égaux devant la loi. Aucune distinction en matière de droits ou de devoirs publics n'intervient entre eux pour des raisons de sexe, d'origine, de langue, de religion ou de foi» (article 28). Religion et traditions ne sont pas oubliées pour autant. L'article 10 fait ainsi de «la famille la base de la société. Elle repose sur la religion, les bonnes mœurs et l'amour de la patrie» et l'article 17 n'est pas exempt d'ambiguïtés en accordant «à la femme palestinienne le droit de participer effectivement à la vie politique, sociale, culturelle et économique. La loi oeuvrera à abolir toutes les entraves qui interdisent le développement de la femme et sa participation à la construction de la famille et de la société en conformité avec les traditions arabes originelles». 
La «Commission de rédaction de la constitution» offre à l'évidence la plus grande place à l'islam et aux deux autres religions du Livre dans leur acception islamique. Les premiers mots de son préambule, lui-même précédé de la basmalla, y sont consacrés : «Confiants en Dieu et croyant en l'importance mondiale de la Palestine, lieu de rencontre des révélations célestes voulu par Dieu [...], les Palestiniens etc.», un lien entre la terre et les «révélations» (al-risâlât) à plusieurs reprises mentionné. L'article 5 fait ainsi de l'islam «la religion officielle de l'État qui [offre] aux révélations célestes respect et sanctification». L'article 6, enfin, stipule que «les principes de la sharia islamique constituent la source principale de la législation. En ce qui concerne les adeptes des révélations célestes, ils bénéficient d'un régime de statut personnel dans la mesure où il s'accorde avec les stipulations de la constitution et la préservation de l'unité, de la pérennité et du développement du peuple palestinien». Dans cette même logique, l'article 38 stipule que «la liberté de foi, de culte et de pratique des rites religieux est garantie dans la mesure où elle ne constitue ni un trouble de l'ordre public ni une insulte pour les révélations célestes».

\section{CONCLUSION}

Chargée de facto de la protection rapprochée d'Israël pour les affaires palestiniennes depuis 1994, l'Ap a été communément jugée à l'aune de son ardeur à réprimer les rangs islamistes. Ses plus ardents ennemis des années 1970 devenus aujourd'hui ses partisans les plus engagés lui ont alors prêté l'idéologie qui fut (ou fut censée être) celle de l'OlP en ces époques où, décidée à libérer la Palestine tout entière, il lui fallait envisager la place qu'elle laisserait aux juifs. Apparu en 1968 et maintenu officiellement comme objectif stratégique de la lutte nationale jusqu'en 1976, «l'État démocratique» avait été conçu avant tout comme «non confessionnel» (lâ-tâ'ifiyya), le Liban étant élevé au rang de repoussoir. La notion avait alors été déclinée en définitions diverses. La coexistence entre musulmans, juifs et chrétiens préconisée par Fath avait été envisagée par les organisations de gauche plutôt en termes de coexistence entre Arabes et Juifs, dans une grande ambiguité toutefois, leur léninisme les obligeant à accorder le droit à l'autodétermination aux Juifs si ceux-ci constituaient un peuple à l'égal des Arabes. Aucun des textes normatifs des grandes organisations nationalistes ni de l'Olp n'avait alors caractérisé cet État de «laïque» (ilmâniyya), notion trop impopulaire car associée le plus souvent à la licence occidentale privée de Dieu. Seuls quelques intellectuels palestiniens (tel Nabîl Chath, alors professeur à l'université américaine de Beyrouth) (Chath, N., 1971) avaient osé le terme, bien vite oubliés ou subrepticement désavoués par les responsables politiques (Yasser Arafat cité dans Gresh. 1983 : 73). Principaux soutiens de la révolution palestinienne en ces années d'anti-colonialisme, les organisations de gauche occidentales avaient alors popularisé un prétendu modèle «laïque» palestinien.

Trente ans plus tard, une distorsion comparable se manifeste à nouveau, au moins implicitement, toujours pour mobiliser un soutien occidental mais sur des objectifs bien différents. Inscrite dans le texte constitutionnel par une commission certes constituée de nationalistes religieux mais pourtant bien représentative des catégories de la population palestinienne (réfugiés et autochtones, anciens cadres de l'Extérieur et leaders de l'Intérieur, etc.), la mention de l'islam comme religion officielle et de la sharia comme source principale de la législation a été de toutes façons approuvée à l'unanimité par le CLP et ne peut donc être rejetée comme fruit d'une sourde manipulation. Cette islamisation des référents politiques palestiniens n'est pas nouvelle. La proclamation de l'État de Palestine en novembre 1988, déjà, était précédée de la basmalla et s'achevait sur un verset coranique. Elle ne fait en réalité que 
traduire l'islamisation de la société elle-même, bien visible depuis bientôt deux décennies déjà par qui voulait la voir et dont Hamas ou le Jihâd islamique étaient loin de rendre compte à eux seuls.

\section{BIBLIOGRAPHIE}

AL-HAQ. 1996. Human Rights in the Proposed Palestinian Basic Law During the Interim Period, Ramallah, Al-Haq.

AL-MARKAZ AL-FILASTÎNî LI-HUQÛQ AL-INSÂN (PCHR). 1999. Al-Majlis Al-Tachrî̀ AlFilastînî. Taqyîm Al-Adâ' Khilâl Dawratay Al-Iniqâd Al-Ulâ wa Al-Thâniyya (Mârs 1996-Mârs 1998) (Le conseil législatif palestinien. Evaluation des performances durant ses première et deuxième saisons (mars 1996-mars 1998), Gaza.

ARURI, N. \& CAROLL, J. 1994. "A New Palestinian Charter”, Journal of Palestine Studies, XXIII/4, Summer 1994, 5-17.

BROWN, H. J. 2000. "Constituting Palestine: The Effort to Write a Basic Law for the Palestinian Authority", Middle East Journal, 54/1, winter, 25-43.

CHATH, N. 1971. "Filastîn al-Ghad" (Palestine de demain), Chu'ûn Filastîniyya, I/2, mai 1971, 5-23.

CHASE, A. 1997. The Palestinian Authority Draft Constitution, Possibilities and Realities in the Search for Alternative Models of State Formation, Jerusalem, Israel/Palestine Center for Research and Information (IPCRI).

CHQÂQ̂̂̀ (Al-), K. (Ed.) 1995. Al-Intikhâbât wa Al-Nidhâm Al-Siyâsî Al-Filastînî (Les élections et le régime politique palestinien), Naplouse, Markaz Al-Buhûth wa Al-Dirâsât Al-Filastîniyya (CPRS), 152-166.

FRIEDMAN, G. 1999. The Palestinian Draft Basic Law, Prospects and Potential, Jérusalem, PICCR, Legal Reports Series 8.

GRESH, A. 1983. OLP. Histoire et stratégies vers l'État palestinien, Paris, Spag-Papyrus.

LEGRAIN, J-F. 1996. "Autonomie palestinienne, la politique des néo-notables", Revue du Monde Musulman et de la Méditerranée (RemmM), 81-82, 1996/3-4, 153-206.

LEGRAIN, J-F. 1998. “Les 1001 successions de Yasser Arafat”, Maghreb-Machrek, n 160, avril-juin, 3-29.

LEGRAIN, J-F. 1999. Les Palestines du quotidien. Les élections de l'autonomie, janvier 1996, Beyrouth, Centre d'Études et de Recherches sur le Moyen-Orient Contemporain (CERMOC).

MAHLER, G. 1996. Constitutionalism and Palestinian Constitutional Development, Jerusalem, Palestinian Academic Society for the Study of International Affairs (PASSIA),

MUNAZZAMAT AL-TAHRÎR AL-FILASTÎNIYYA (OLP). Sd. Qarâr Inchâ' Al-Sulta Al-Wataniyya Al-Filastîniyya (Décision de création de l'Autorité nationale palestinienne), Sl..

NAUFAL, M. 1995. “La crise entre l'Autorité nationale et l'OLP”, Revue d'Études palestiniennes, n 4, nouvelle série, été 1995, 3-16.

QASEM (Al-), A. 1992-1994. "Commentary on Draft Basic Law for the National Authority for the Transitional Period", The Palestine Yearbook of International Law, 1992/1994, 187-211.

QASEM (Al-), A. 1996. "The Draft Basic Law for the Palestinian National Authority during the Transitional Period". In: E. Cotran and C. Mallat (eds.) 1996. The Arab-Israeli Accords: Legal Perspectives. London, Kluwer Law international, 101-156.

SHEHADEH, R. 1997. From Occupation to Interim Accords, Israel and the Palestinian Territories, La Haye, Kluwer Law international. 
WING, A. 1994. Democracy, Constitutionalism and the Future State of Palestine, with a Case Study of Women's Rights, Jerusalem, PASSIA.

WING, A. 1999. "The Palestinian Basic Law, Embryonic Constitutionalism", Case Western Reserve Journal of International Law, XXXI/2-3, Spring/Summer 1999 accessible sur http://129.22.185.151/ jilweb/31-2.htm.

\section{NOTES}

1 L'original arabe, qui compte 97 articles sous 7 têtes de chapitre, a été publié privé de son chapeau in Al-Nahâr, Jérusalem, 12 juin 1994. Une version anglaise non officielle est reproduite in Palestine Report, 7/1, 1 er janvier 1994 (un décrochement dans la numérotation des articles s'opère entre l' arabe et l'anglais à partir de l'article 25 sans impact sur le contenu du texte. La version du Jerusalem Report compte donc 98 articles. Ce draft prévoit la promulgation du texte pour le 13 décembre 1993. Le texte de la «Commission de rédaction de la constitution» sera le seul autre projet mentionnant une date de promulgation : le 13 septembre 2000.

2 L'original arabe, qui compte 106 articles sous 7 têtes de chapitre, est reproduit in Al-Nahâr, 5 février 1994 et Al-Quds, Jérusalem, 6 février 1994. Il est analysé dans AruRi N. \& CARoLl J., «The Palestinian 'Constitution' and the 'Old Regime'», Middle East International, 15 avril 1994.

3 L'original arabe, qui compte 108 articles sous 6 têtes de chapitre, et sa traduction anglaise ont été publiés par le JMCC, «Occasional document series», $\mathrm{n}^{\circ} 1$, juin 1994, la traduction anglaise étant disponible sur le site du JMCC (http://www.jmcc.org/research/series/basicl.html). Une traduction anglaise, fournie par Anîs Al-Qâsim lui-même, est publiée in The Palestine Yearbook of International Law, 1992/1994, 171-186 de même que dans QASEM (AL-), A. 1996. L'original arabe est reproduit in CHQÂQî (AL-), K. (Ed.) 1995, 152-166. Une traduction anglaise est également reproduite in Journal of Palestine Studies, XXIII/4, Summer 1994, 137-145. Une analyse générale du texte en comparaison avec les précédentes versions est proposée in ARURI, N. \& CAROLL, J. 1994. Une autre analyse comparative, centrée cette fois sur la question des droits de la femme, est menée in WING, A. 1994. Lire également WiNG, A. 1999.

4 L'original arabe, qui compte 122 articles sous 7 têtes de chapitre, et sa traduction anglaise ont été publiées par le JMCC, «Occasional document series», $\mathrm{n}^{\circ} 5$, février 1996. Des traductions anglaises sont reproduites in Palestine Report, 9 février 1996 (disponible sur le site du JMCc http://www.jmcc.org/ research/series/basic2.htm), et in MAHLER, G. 1996, 344-357, qui mène également une analyse comparative avec les versions antérieures.

5 MAHLER, G. 1996, 135 lui accorde le numéro 4-a. Lire AL-HAQ. 1996. Ce texte a lui-même connu 2 drafts principaux (l'un de 154 articles, l'autre de 159 sous 8 têtes de chapitre), l'ultime étant daté du 27 mai. Sa mise en ligne est annoncée sur le site du centre (http://lawcenter.birzeit.edu). Je tiens ici à remercier Bernard Botiveau et Genia Helou-Raad, du Centre de droit, pour leur aide documentaire.

6 Al-Markaz Al-Filastîîi li-HuqùQ Al-Insân (PChr). 1999, 41-54, retrace les étapes du processus d'adoption de la loi par le ClP.

7 Il semblerait que lesélus de Cisjordanie n' aient pu que très rarement participer aux réunions du Comité tenues à Gaza.

8 Le texte est doté de 133 articles sous 7 têtes de chapitre et a été publié dans la presse palestinienne le 16 juin 1996. Cette version semble correspondre à la «version zéro» de la chronologie tenue par le CLP.

9 Doté de 125 articles réunis sous 6 têtes de chapitre, le texte a été achevé 14 juillet 1996

10 Dans les publications officielles du CLP, le projet examiné, amendé et voté est pourtant attribué «à l'initiative du ministère de la Justice». 
1 CHASE, A. 1997, 36 mentionne ces 2 textes mais s'abstient de leur accorder un numéro après les avoir jugés avoir été dépourvus de tout impact. Je n'ai pu me procurer le texte de Naplouse. Je tiens à remercier Dr Ali Khashan d'avoir bien voulu me communiquer le texte inédit du projet.

12 Cette version semble correspondre à la « ${ }^{\text {ère }}$ version» de la chronologie du CLP. Il s'agit du draft analysé par CHASE, A. 1997. qui lui octroie le numéro 8

13 Une traduction anglaise de cette version dotée de 112 articles sous 6 têtes de chapitre était disponible sur le site du CLP (http://www.pal-plc.org) lorsqu'il fonctionnait en 1998. On la trouve également sur le site de Palestine Online (http://www.p-ol.com/highway/government/basiclaw.htm ou http:// www.ramallahgrandpark.com/highway/government/basiclaw.htm) ou encore sur le site de l'Administrative Improvement Unit de l'Autorité (http://www.pna-aiu-pank.org/network/net8.html)

14 Une traduction anglaise de cette version dotée de 112 articles en 7 chapitres est disponible sur le site de LAw (http://www.lawsociety.org). FrIEDMAN. G. 1999, prend cette version pour base de son analyse.

15 Selon Anwar Abu Eisheh, professeur à la Jerusalem 's Palestinian School of Law de l'université AlQuds, que je remercie pour toute son aide.

16 Il n'aurait cependant jamais participé aux travaux de cette commission.

17 Al-Ayyâm, 4 juillet 2000. Le texte est alors censé compter 165 articles sous 6 têtes de chapitre avec un préambule.

18 Le texte compte 181 articles sous 6 têtes de chapitre avec un préambule.

19 Peut-être se situe-t-il dans la lignée du projet d'Al-Najâh dont je n' ai pu me procurer copie ? Différent par les termes et l'ordonnancement des articles, il l'est également par ses dispositifs institutionnels. A la différence, par exemple, des textes antérieurs qui ne mettaient en place qu'une seule assemblée législative, ce texte prévoit un système bicaméral avec un Conseil national élu par les citoyens de l'Etat de Palestine et par la diaspora et un Conseil législatif formé de députés élus par les seuls citoyens de l'Etat (article 59 et suivants). Au détour d'un article (110), il fait également apparaître une notion absente partout ailleurs : «Le président de l'Etat est le président de la République».

20 Al-Ayyâm, 16 septembre 2000, fait état de ce texte qui inclurait la proclamation de l'indépendance de 1988 et comprendrait 3 chapitres de 41 articles.

${ }^{21}$ Peut-être se situe-t-il dans la lignée du projet d'Al-Najâh dont je n'ai pu me procurer copie ? Différent par les termes et l'ordonnancement des articles, il l'est également par ses dispositifs institutionnels. A la différence, par exemple, des textes antérieurs qui ne mettaient en place qu'une seule assemblée législative, ce texte prévoit un système bicaméral avec un Conseil national élu par les citoyens de l'Etat de Palestine et par la diaspora et un Conseil législatif formé de députés élus par les seuls citoyens de l'Etat (article 59 et suivants). Au détour d'un article (110), il fait également apparaître une notion absente partout ailleurs : «Le président de l'Etat est le président de la république».

22 La même formule figure le plus souvent dans les en-têtes officielles des «ministères», sur les enveloppes, le papier à lettres, etc. Ainsi, si le draft élaboré par le ministère de la Justice ignore la basmalla, l'en-tête du ministère qui le surmonte l'inclut.

23 En l'absence de précision contraire, les articles mentionnés sont identiques et portent le même numéro dans l'ensemble des versions produites par le CLP.

24 Le 2 août 1994, pourtant, Yasser Arafat nommait Ibrâhîm Dughma à la tête d'un «département de la fatwa et de la législation», département officiellement créé le 9 décembre 1995 (décret 286/1995), selon SHEHADEH, R. 1997, 147.

25 A force d'amender leur texte, les législateurs commettent des fautes de grammaire, le pluriel employé pour les 3 religions célestes n'est plus de mise dès lors qu'il s'agit dorénavant des 2 religions célestes autres que l'islam. 